Version of April 13, 2017

\title{
ROTATIONAL AND CYCLICAL VARIABILITY IN $\gamma$ CASSIOPEIAE. II. FIFTEEN SEASONS
}

\author{
GREGORY W. HENRY \\ Center of Excellence in Information Systems, \\ Tennessee State University, \\ 3500 John A. Merritt Blvd., Box 9501, Nashville, TN 37209; \\ gregory.w.henry@gmail.com \\ and \\ MYRON A. SMITH \\ Catholic University of America, \\ 3700 San Martin Dr., Baltimore, MD 21218; \\ msmith@stsci.edu
}

\begin{abstract}
The B0.5 IVe star $\gamma$ Cas is of great interest because it is the prototype of a small group of classical Be stars having hard X-ray emission of unknown origin. We discuss results from ongoing $B$ and $V$ observations of the $\gamma$ Cas star-disk system acquired with an Automated Photometric Telescope during the observing seasons 1997-2011. In an earlier study, Smith, Henry, \& Vishniac showed that light variations in $\gamma$ Cas are dominated by a series of comparatively prominent cycles with amplitudes of $0.02-0.03$ mag and lengths of 2-3 months, superimposed on a 1.21-day periodic signal some five times smaller, which they attributed to rotation. The cycle lengths clustered around 70 days, with a total range of 50-91 days. Changes in both cycle length and amplitude were observed from year to year. These authors also found the $V$-band cycles to be 30-40\% larger than the $B$-band cycles. In the present study we find continued evidence for these variability patterns and for the bimodal distribution of the $\Delta B / \Delta V$ amplitude ratios in the long cycles. During the 2010 observing season, $\gamma$ Cas underwent a mass loss event ("outburst"), as evidenced by the brightening and reddening seen in our new photometry. This episode coincided with a waning of the amplitude in the ongoing cycle. The Be outburst ended the following year, and the light-curve
\end{abstract}


amplitude returned to pre-outburst levels. This behavior reinforces the interpretation that cycles arise from a global disk instability. We have determined a more precise value of the rotation period, $1.215811 \pm 0.000030$ days, using the longer 15-season dataset and combining solutions from the $V$ and $B$ light curves. Remarkably, we also find that both the amplitude and the asymmetry of the rotational waveform changed over the years. We review arguments for this modulation arising from transits of a surface magnetic disturbance. Finally, to a limit of $5 \mathrm{mmag}$, we find no evidence for any photometric variation corresponding to the $\gamma$ Cas binary period, 203.55 days, or to the first few harmonics.

Subject headings: circumstellar matter -stars: individual ( $\gamma$ Cas $)$-stars: emissionline, Be

\section{Introduction}

$\gamma$ Cas (HD 5394, B0.5 IVe) is the prototype of the classical Be stars, defined as nonsupergiant, non-pre-main-sequence B stars with Balmer-line emission recorded sometime in their history but without ongoing mass infall. The Balmer-line emission arises from a flattened Keplerian disk. Be stars have broad photospheric lines due to their rapid rotation (e.g., Porter \& Rivinius 2003). $\gamma$ Cas was the first star with $\mathrm{H} \alpha$ emission to be reported (Secchi 1866). It became important again with the discovery of its copious hard X-ray emission (Jernigan 1976), which is unusual for early-type stars (White et al. 1982; Güdel \& Nazé 2009). Its high-energy peculiarities include an X-ray light curve that varies on several timescales ranging from seconds to 2-3 months (Smith, Robinson, \& Corbet 1998, hereafter SRC98; Robinson, Smith, \& Henry 2002, hereafter "RSH"), a luminosity $\mathrm{L}_{X_{2-10}}$ $\sim 10^{33} \operatorname{ergs~s}^{-1}$, and an X-ray spectrum that reveals the presence of 3-4 optically thin hot plasma components. $\gamma$ Cas is now one of a group of at least nine recognized Galactic X-ray emitting B0.5e-B1.5e stars (Lopes de Oliveira et al. 2006; Smith et al. 2012, hereafter $\mathrm{S} 12)$. The source of the X-rays remains a source of spirited debate. The two most common suggestions are that the emission comes from accretion from the Be star wind or disk (White et al. 1982) or from the interaction of magnetic field lines from the star and its decretion (mass loss) disk, with an ensuing release of high-velocity particle streams impacting the Be star and causing explosive high-energy events, often dubbed "flares" (see Robinson \& Smith 2000, hereafter RS). In the latter scenario, flares are not necessarily products of local magnetic instabilities, such as solar-type flares. The difficulty in settling the controversy comes from the fact that the X-rays can arise, in principal, from any environment in which particles (electrons or protons) can be accelerated to high velocities, either by release of 
tension in stretched magnetic field lines or in a steep gravitational potential. Probably the most telling piece of evidence lies in the simultaneous rapid variations of X-ray flux and UV and/or optical signatures that are presumed to form close to the Be star (e.g., Slettebak \& Snow 1978; Peters 1982). To get a handle on this, SRC98 launched a 21-hour campaign on $\gamma$ Cas that included X-ray observations by the Rossi X-ray Timing Explorer (RXTE) and spectroscopic and photometric observations by the Hubble Space Telescope (HST). The latter component was a very high time-resolution monitoring of the Si IV 1394-1403 A resonance line doublet and a surrounding quasi continuum. The latter could be binned in wavelength to give a time record of both the UV continuum and several lines that were formed in the photosphere. The UV continuum record showed a pair of faint dips that were investigated by Smith, Robinson \& Hatzes (1998), who demonstrated that these absorption features arose from cool, translucent clouds that transit the star. These structures transited too rapidly to be surface features, nor could they easily be due to structures within the Keplerian disk because Long Baseline Optical Imaging has demonstrated that the disk plane is tilted by about $48 \pm 4^{\circ}$ with respect to our line of sight to the star (e.g., Stee et al. 2012). These same authors noted similar dip features several days later in spectra from International Ultraviolet Explorer (IUE). The UV spectra evinced narrow components moving blue-to-red across the line profiles, consistent with optical spectra of other observers (e.g., Yang, Ninkov, \& Walker 1988; Smith 1995). These so-called migrating subfeatures ( $m s f$ ) have also been observed in a He I line of another $\gamma$ Cas star, HD 110432 (Smith \& Balona 1998). Such features have long been observed in the magnetically active pre-main-sequence K5e star, AB Dor (CameronCollier \& Robinson 1989).

$\gamma$ Cas is now known to be the primary star of a wide binary system with $\mathrm{P}=203.5 \pm 0.20$ days (Harmanec et al. 2000; Miroschnichenko et al. 2001; Nemravová et al. 2012; S12). The last three of the cited studies agree that the binary system is nearly circular $(e<$ $0.03)$, and the unseen secondary has a mass of $\frac{1}{2}-1 \mathrm{M}_{\odot}$. At least two " $\gamma$ Cas analogs" are blue stragglers, according to their likely memberships in galactic clusters (e.g., Marco et al. 2009). Conceivably all of them are blue stragglers, although this is not yet well established. Moreover, no evidence exists for $\gamma$ Cas being a blue straggler since it is not known to be associated with a galactic cluster. Aside from the obvious possibility that the secondaries of such stars could accrete matter from the Be star, it is also possible that their previous evolutionary history could have resulted in mass and angular momentum transfer to the current primary, thereby spinning it up. The fact that $\gamma$ Cas rotates close to its critical velocity may be part of what is needed for the X-ray generation mechanism to develop.

Although $\gamma$ Cas has been observed by nearly all X-ray space telescopes, its brightness ( $V$ $\approx 2.1$ ) makes it inaccessible to most modern UV and optical instruments because it saturates their detectors. However, with suitable use of neutral density filters, $\gamma$ Cas has provided an 
excellent target for monitoring with the T3 0.4m Automatic Photometric Telescope (APT) in Arizona (see $\S 2$ below). Ironically, long-term optical monitoring by this small, ground-based telescope has resulted in a series of major discoveries, some or all of which may turn out to have bearing on the X-ray emission mechanism, which orbiting X-ray telescopes themselves could not elucidate. These include:

- the presence of a robust period of 1.21 days in optical flux (Smith, Henry, \& Vishniac 2006, hereafter SHV) with an unusual waveform. There is no detectable variation in the $B-V$ color index at this period, suggesting the variation is due to rotational advection of a surface feature. We will address the rotational interpretation of this feature in $\$ 4.2$.

- the existence of long optical cycles of 50-91 days, which seem to correlate with corresponding X-ray cycles (RSH; SHV). These short-lived cycles have larger amplitudes in the Johnson $V$ band than in the $B$ band. Because the variations are "red," they probably arise in the Be disk, which contributes $10-50 \%$ of the system's integrated light at visible wavelengths. This percentage increases rapidly in the infrared, where at least the inner disk becomes optically thick because of hydrogen's dominant bound-free and free-free transitions.

- a brightening and reddening of the star-disk system in 2010, reaching a peak in early 2011, then fading through the end of our dataset (2012 February 24). There was a corresponding increase in $\mathrm{H} \alpha$ emission (S12), and we refer to this extended event as the "2010 outburst." The outburst was also correlated with the second and longest of a two-density column system observed in contemporaneous (2010 July-August) highresolution X-ray spectra. The increase in this column is manifested by a decrease in soft X-ray band flux $\left(\frac{1}{2}-1 \mathrm{keV}\right)$ relative to flux in the hard band $(2-8 \mathrm{keV})$, as in all previous epochs for which these fluxes are recorded (S12).

This paper presents the results of our ongoing photometric monitoring campaign on $\gamma$ Cas. SHV covered observing Seasons 1997-2004. This paper extends the time coverage through the 2011 observing season.1 Our observations include both the 2010 outburst and also the next season during which the brightness declined, though not yet to its level at the start of the outburst. We analyze the long cycles in the new seasons, including their color

\footnotetext{
${ }^{1} \gamma$ Cas comes to opposition in early October, and we define the $\gamma$ Cas observing seasons, e.g., the "2004" observing season, as the time interval 2004 May-2005 February, minus the months of July and August lost to the monsoon rains that come to southern Arizona every summer.
} 
variations, and search for additional long cycles. We also reanalyze the long-cycle subtracted residuals to better define the 1.21 day variation and its behavior with time.

\section{The Automated Photometric Telescope Observations}

Our optical continuum observations of $\gamma$ Cas were acquired with the T3 $0.4 \mathrm{~m}$ Automated Photometric Telescope (APT) at Fairborn Observatory, located in the Patagonia Mountains of southern Arizona. T3 is one of eight Tennessee State University (TSU) telescopes operated at Fairborn for automated photometry, imaging, and spectroscopy (Eaton, Henry, \& Fekel 2003; Eaton \& Williamson 2007).

Full details concerning our $\gamma$ Cas observing program can be found in RSH and SHV, and details of telescope operations and data reduction procedures can be found in Henry (1995a, 1995b). Briefly, the T3 APT was programmed to observe $\gamma$ Cas once every two hours on every clear night throughout its observing season. For each observation, the APT cycles between $\gamma$ Cas and the nearby comparison and check stars HD 6210 (F6 V) and HD 5395 (G8 IIIb), respectively, in the following sequence, termed a group observation: $\mathrm{K}, \mathrm{S}, \mathrm{C}, \mathrm{V}, \mathrm{C}, \mathrm{V}, \mathrm{C}, \mathrm{V}$, $\mathrm{C}, \mathrm{S}, \mathrm{K}$, where $\mathrm{K} \& \mathrm{C}$ are the check and comparison stars, $\mathrm{V}$ is $\gamma \mathrm{Cas}$, and $\mathrm{S}$ is a sky reading.

Each $\gamma$ Cas measurement is thus bracketed by the comparison star on both sides three times during each group observation. All observations were made in the standard Johnson $B$ and $V$ passbands with 10 s integrations for $\gamma$ Cas and HD 5395 and 20 s for HD 6210 and the sky readings. In addition, all measurements (except for the 1997 observing season) were made through a 3.8 mag neutral density filter to attenuate the counts from $\gamma$ Cas and so avoid large deadtime corrections. However, as noted in SHV, a different neutral density filter was used for the 1997 season from the more permanent one we selected in 1998, and thus the 1997 season has not been used in those analyses requiring linkage among the seasons. A complete sequence, including both $B$ and $V$ filters, requires about 8 minutes to execute. Group mean $V-C$ and $K-C$ differential magnitudes are computed and standardized with nightly extinction and yearly-mean transformation coefficients determined from nightly observations of a network of standard stars. Group means are taken to be single observations thereafter. Typical rms errors for a single observation, as measured from pairs of constant stars, are $\pm 0.003-0.004$ mag. The 15 yearly-mean $K-C$ differential magnitudes scatter about their grand mean with an rms of only 0.0019 mag, indicating very good long-term stability in the brightness of both the comparison and check stars as well as good stability of our ND filters and calibrations to the Johnson photometric system.

The $V-C$ differential magnitudes were converted to apparent magnitudes of $\gamma$ Cas by assuming apparent magnitudes $\mathrm{m}_{V}=5.84$ and $\mathrm{m}_{B}=6.40$ for the comparison star. Aside from 
our inability to properly calibrate the 1997 season with the data from subsequent seasons, our seasonal-mean brightness values should have an internal precision of approximately \pm 0.002 mag, as shown by the seasonal mean $K-C$ values, while the ND filter limits the accuracy of our apparent magnitudes to $1-2 \%$.

SHV presented $3135 \mathrm{~V}$ and $3157 \mathrm{~B}$ observations covering the nine observing Seasons 1997-2005. In 1998 the filter was changed to another, which has been used in the meantime. Here, we present data from six more observing seasons 2006-2011, for a total of $4675 \mathrm{~V}$ and 4716 B observations. All 15 years of data (1997-2011) are listed in Table 1, available in the online version of this paper.

Table 1 : Photometric Observations of $\gamma$ Cas in Seasons 2006-2011

\begin{tabular}{c|c|c|c|c}
\hline $\begin{array}{c}\text { Reduced Julian Date } \\
\text { RJD }\end{array}$ & $\begin{array}{c}\text { Var } B \\
(\mathrm{mag})\end{array}$ & $\begin{array}{c}\text { Var } V \\
(\mathrm{mag})\end{array}$ & $\begin{array}{c}\text { Chk } B \\
(\mathrm{mag})\end{array}$ & $\begin{array}{c}\text { Chk } V \\
(\mathrm{mag})\end{array}$ \\
\hline 50718.6966 & -4.393 & -3.641 & -0.818 & -1.216 \\
50718.9253 & -4.393 & -3.642 & -0.814 & -1.222 \\
50720.7936 & -4.386 & -3.644 & -0.821 & -1.218 \\
50720.9191 & -4.387 & -3.638 & 99.999 & -1.223 \\
50721.6940 & 99.999 & -3.648 & 99.999 & 99.999 \\
50721.7933 & -4.386 & -3.639 & -0.816 & -1.212 \\
\hline
\end{tabular}

Note. - Table 1 is presented in its entirety in the electronic edition of the Astrophysical Journal. A portion is shown here for guidance in data format and content.

Note. - An entry of 99.999 signifies that the differential magnitude was discarded because its internal standard deviation exceeded $0.01 \mathrm{mag}$, indicating nonphotometric conditions.

\section{RESULTS}

\section{1. $\quad$ Long-Term Brightness and Color Variations}

In Figure1, we present the seasonal mean $V$ magnitudes and $B-V$ color indices for the 1998-2011 observing seasons. The figure shows that the light from the Be star-disk system brightened and reddened during most of this interval, in fact by a total of about $5 \%$. As noted by S12, half of the brightening and reddening increases started in mid-2010, fortuitously coinciding with a simultaneous campaign of XMM-Newton and Long Baseline 
Optical Interferometric observations. This event, an example of what is referred to as an "outburst" of a classical Be star, reached peak brightness shortly before APT observations resumed on 2011 June 12, the last observing season analyzed in this paper.

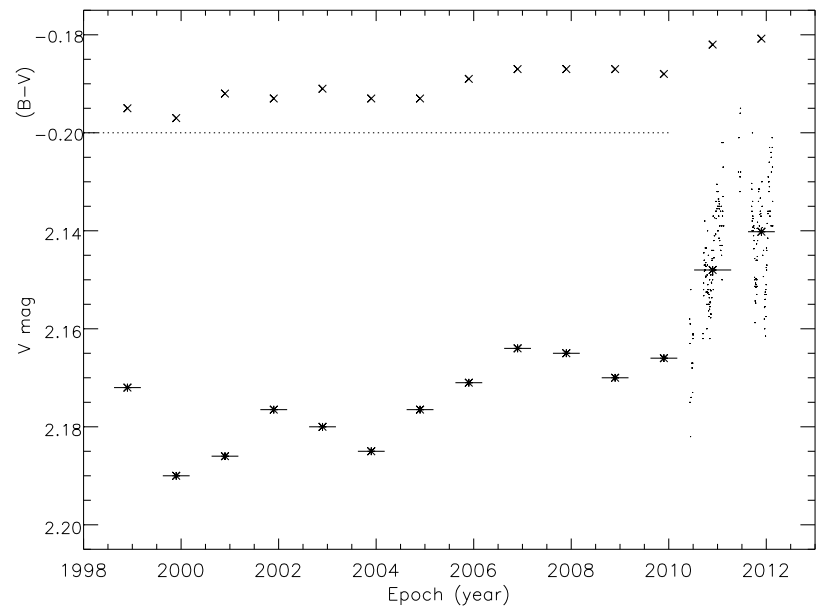

Fig. 1.- Johnson $V\left(\mathrm{~m}_{V}\right)$ magnitudes (* symbols) and $(B-V)$ color indices (crosses) of $\gamma$ Cas acquired with the T3 APT at Fairborn Observatory during the 1998-2011 observing seasons; large symbols represent seasonal means. Error bars on the means are smaller than the symbol size. Horizontal bars denote the coverage for a seasonal mean time. Small dots indicate individual $\mathrm{m}_{V}$ measurements in the 2010 and 2011 seasons. All magnitudes in this paper are plotted with brightness increasing upward.

Although brightness variations in $B$ and $V$ are highly correlated, the flux recorded in the $V$ band is more sensitive to brightening events than $B$, implying the activity is concentrated in the disk. Following SHV, we parameterize this by computing for each season a magnitude range ratio $r=\Delta B / \Delta V$, where $\Delta B$ and $\Delta V$ are computed as the means of the differences of the absolute values of each observation in a season with respect to the seasonal mean values, $\langle B\rangle$ and $\langle V\rangle$. So defined, the $r$ index tends to unity for variations in the photosphere and to values of 0.5-0.7 for structural changes in the disks of Be stars in general and of $\gamma$ Cas in particular (Harmanec et al. 1980; Hirata 1982; Barylak \& Doazan 1986; Hirata 1995). Figure2 exhibits the regression line formed from the 1998-2009 seasons. The correlation of $r$ with system brightness $V$ is marginally significant (at $2.5 \sigma$ ) and would be lower with the addition of the last two seasons. In Fig.2 the symbols for these two seasons are connected by a dashed line, indicating the $r / V$ trajectory during the outburst. The brightening observed during this period can be interpreted as a simultaneous injection of mass from the star to the disk. (Of course the attenuation of the soft X-rays indicates that the expelled gas also occupies a larger volume that includes our sight line to the star.) Within the errors, neither the $r$ value nor the period of the long cycle seems to have been affected by the outburst. 


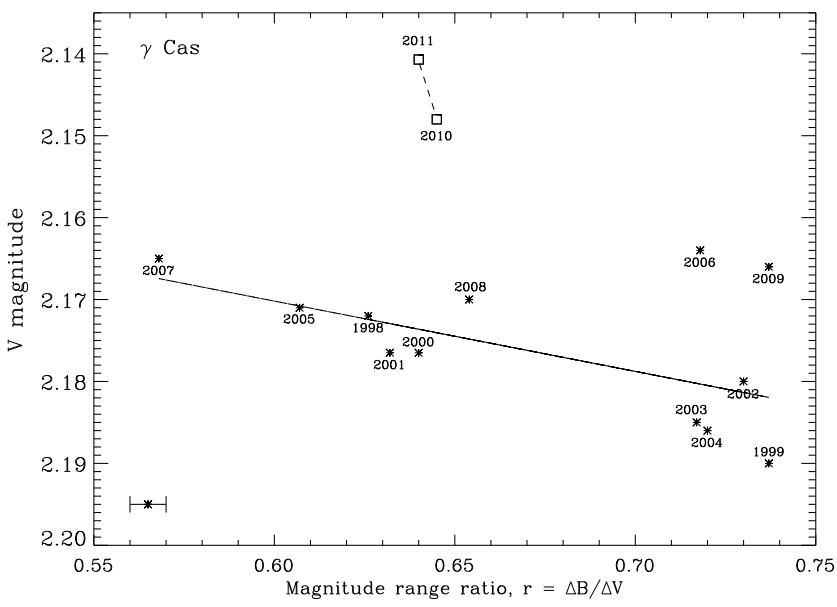

Fig. 2.- The seasonal quantities $r=\Delta B / \Delta V$ discussed in the text plotted against seasonal $V$ means for $\gamma$ Cas. For each of the two filters, the " $\Delta$ " quantity is the mean of the absolute values of all observations in a season with respect to the seasonal average. Season annotations are indicated near each plotted point. The solid line is the regression fit through the points, except for of the 2010 outburst \& 2011 decline intervals.

In their study of light curve averages for seasons 1997-2005, SHV tentatively concluded that the values of the seasonal $r$ ratios tend to clump around two values, near 0.62 and near 0.72, with a gap between them. The additional seasons in this study, shown in Figure 3 , tend to confirm the impression of two separated groups. A Monte Carlo analysis of an assumed distribution of these seasonal amplitude ratios shows that the gap is marginally significant (to $2 \sigma$ ). In this exercise our bin sizes, were 0.01 magnitudes, which is closely matched to the rms of \pm 0.009 measured from the data subsets from which the r values were computed. This implies that the amplitude ratio $r$ has a weak dependence on its recent history - that is, what may be important is the question: has the disk been bright or faint in recent times?

\subsection{The Long-Term Cycles}

RSH and SHV found that the long cycles of $\sim 70$ days are the dominant source of brightness variation in the optical light curve of $\gamma$ Cas. For some seasons SHV were able to fit these variations well in both $B$ and $V$ with a single sinusoid. In these cases the brightness variation could be fit with four parameters: slope, period (cycle length), amplitude, and mean brightness level of the sinusoid. However, for most seasons in the current study, we had to adopt a search for "am/fm"-type modulations, that is, we were usually forced to consider linear changes with time in the amplitude $(\mathrm{am})$ and/or length $(\mathrm{fm})$ of our sinusoids 


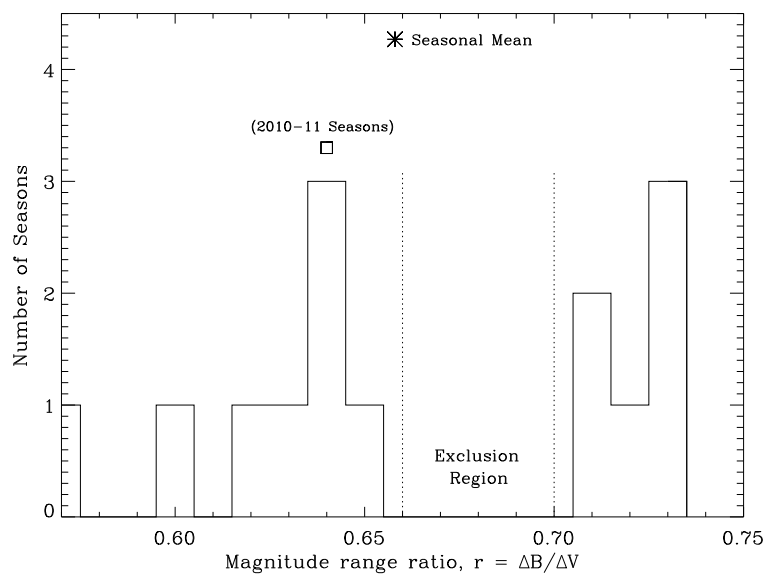

Fig. 3.- Histogram of the $\mathrm{r}$ values (defined as in Fig.22). To a modest level of significance the distribution is bimodal. The mean $r$ for all seasons, except the anomalous Seasons 2010 and 2011, is shown by an asterisk. The mean value of the last two seasons is labeled separately.

to obtain good fits. Often a mild linear trend had to be imposed throughout a whole season. The data were uncorrected for the 1.21-day rotational period discussed in the next section. This signal is typically five and sometimes ten times smaller than the cycle amplitudes.

We used the Interactive Data Language to develop customized procedures for periodogram fitting the long cycles to the observations in each observing season. We determined "best fits" to the slope, cycle length, amplitude, and mean brightness with the MPFIT package published by Markwardt (2011). However, a simple sinusoid-fitting procedure gave a reasonable solution for only about half the seasons. As noted by RSH and SHV, the long cycles exhibit changes in all four parameters. Sometimes it was possible to link one season to the next with only small changes to these parameters, e.g., the $2006 \& 2007$ and also the 2010 \& 2011 seasons. In these two cases, only small changes in the slope and cycle length parameters, (within the formal uncertainties of the Markwarth solution) were needed to link the two seasons. In other cases, it is debatable how the cycle lengths and amplitudes change with time. We also note that the time interval between seasons is $90 \pm 10$ days. Therefore, we discarded this simple procedure in deference to economy of assumptions. In most cases, our next step was to seek a solution linking roughly the later two thirds of one season with the initial two thirds of the next. Sometimes, even this procedure failed to give satisfactory fits because, as found by SHV for Seasons 1998 and 2003, a particular cycle could damp out in the interim. The cycle usually recovered within weeks but sometimes with different parameters. SHV reported that in Season 2003 this occurred with a recovery of the former 
cycle's phase. Because of these characteristics, we minimized the number of parameters by requiring that a new oscillation grow from an old one with the same phase at the null point. Given the variety of behaviors, it is impossible to claim that the fits are unique because their qualitative characteristics can be quite different. However, we are confident that alternative solutions are either worse or require more free parameters. By fitting the $B$-band data over the same timespan as the $V$ solutions and comparing best-fit parameters, we estimate typical errors in the cycle lengths to be about \pm 1 day, while the amplitudes are estimated to be uncertain by $10-15 \%$. These estimates must be tempered by the uncertainty of whether or not the long-term trends in the light curves vary linearly, i.e., free of other system noise sources, such as irregular injections of mass into the disk.

Figures 4 and 5 depict our best-fit solutions to the nightly means of Seasons 20052011. Each panel in Fig. 4 shows the solution for each season changing smoothly into the solution of the next. Therefore, data in the middle of a particular season reflect a continuous solution from the previous to the next season. The quality of the solutions is similar to those discovered by SHV.

The Season 2006 data suggest a cycle length of $~ 93$ days, while Season 2008 suggests the growth of a new, rather long cycle, perhaps $\sim 100$ days in length. These cycle lengths are slightly longer than the 91 day length that SHV encountered for Season 2000. The variations in Season 2007 alone suggest a solution that can be extrapolated to much of the next season. Even so, the brightness during the beginning of this season (RJD $=54260)$ suggests that the star-disk system was abnormally faint. In particular, Season 2005 exhibits a damped oscillation that grew to a new cycle in 2006, with no evident change in phase. In the first part of Season 2009 a new cycle has started from the previous season. In fact, we suspect that the matter injection into the disk was not regular either. This would account for the unusually poor fit at this time. From the inadequate fit at RJD $=55100-55150$ in Season 2009 , we suspect that a new cycle started growing in this interval. Unfortunately, inclement weather in 2009 June meant we could not determine what the trend in the variations were at this critical time. For the other seasons, the complexity of the parameterization invoked for the fits is not a fault of inadequate time coverage or noisy data. This can be verified by independent solutions for the $B$ band, which show similar details in the departures from the data.

The two least satisfactory fits were during times of anomalous behavior of the light curve in Seasons 2005 and 2010 - see Fig.4. The first of these was during yet another damping of an old cycle (period) and growth of a new one. The brightness decreased during this interval. The other inadequate fit occurs in the middle of Season 2010 during the growth of the 2010 outburst. Again, this flaw may be related to the photometric/spectroscopic outburst during 

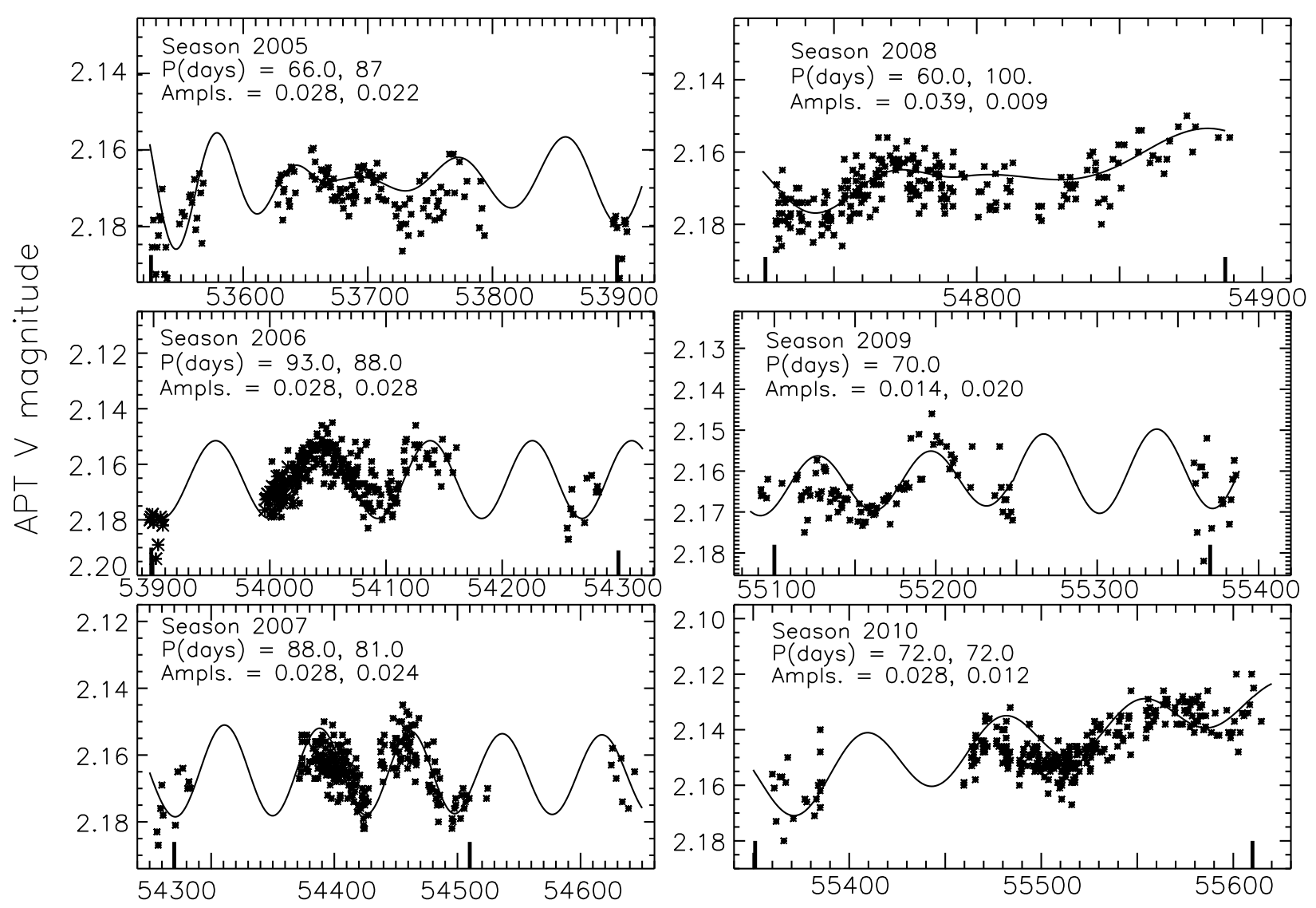

Time (HJD - 2400000)

Fig. 4.- $V$-band photometry of $\gamma$ Cas showing the light curve during Seasons 2005-2010, ending in 2011 February. The approximate date of the immediate post-maximum of 20102011 is indicated by a small clump of points at 2011.5. The solid line indicates the damped or changing sinusoids needed to fit the data. Their combs at the bottom of the panels show the reference start and end times for the computation of these sinusoids using the periods and amplitudes indicated.

this time. We also notice from Fig. [5 that not only did the cycle persist but its amplitude grew to a more typical value after the event ceased. The restored amplitude is sufficiently large that the oscillation can be discerned in the $B-V$ color index during this interval.

For the 2005-2011 observing seasons analyzed in this paper all cycle lengths fall within the same range of 50-93 days observed by SHV for Seasons 1997-2004, where again the upper limit may be 100 days. The means and rms values of the cycle lengths are $76 \pm 11$ day. Fully $20 \%$ of the cycle lengths deviate by $\geq 20 \%$ from this mean. Like SHV, we find the 


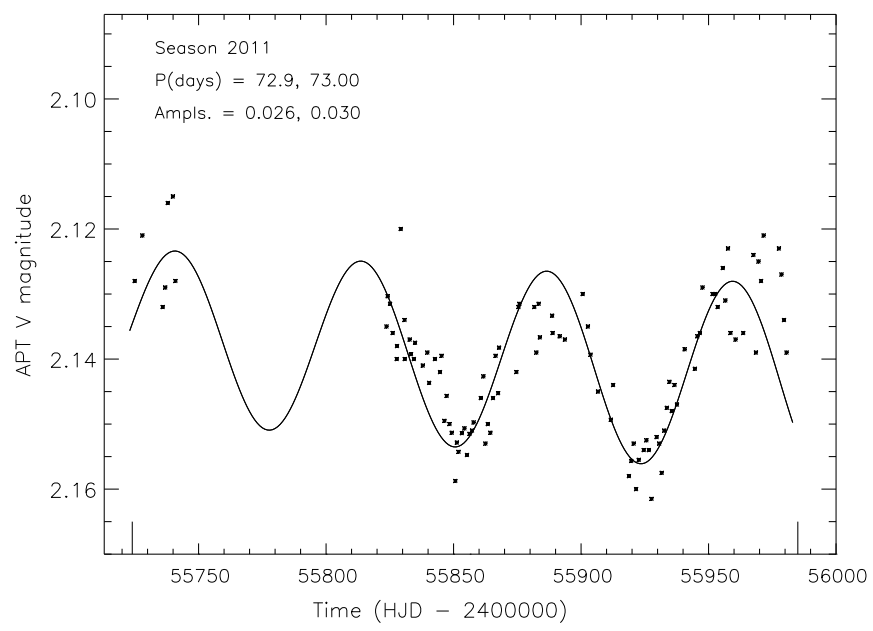

Fig. 5.- $V$-band photometry of $\gamma$ Cas showing the long cycles of Season 2011. Note the trend to fainter magnitudes after reaching a maximum in mid-2011 following the 2010 "outburst."

cycle amplitudes in the $V$ band photometry to be 0.006-0.030 magnitudes.

\subsection{The 1.21-day periodicity}

The extraction of the 1.21 day signal found by SHV requires some caution, first because the period is near one day and second because the amplitude is nearly an order of magnitude smaller than the dominant long-cycle variation, which, according to SHV, is nonsinusoidal. We began by detrending each observing season of the $V$ and $B$ datasets and forcing each to a common mean brightness. We ignored obvious low-frequency peaks caused by the long cycles, and this allowed us to isolate a smaller peak at 0.8225 day $^{-1}$ that was found and identified as a rotational signature. In the time domain, this resulted in a predicted sinusoidal variation for the entire dataset that we refer to as the multiseasonal average.

SHV noted the possibility that the seasonal mean amplitudes and even phases of this periodicity might vary, but they did not pursue the question further. With the extended timeline of six more seasons, we decided to examine this possibility by imposing the ephemeris (period and phase of mininum light for the multiseason solution) and searching for variations in the phases and amplitudes in the individual seasonal datasets. In fact, we found substantial variations in both amplitude and reference phase from season to season. The full amplitudes ranged from 4-8 mmag prior to 2004 but ranged only between 1-3 mmag from 2005 onward.

To see whether these amplitude changes are caused by subtle effects from the dominant, 
long-period signals, we prewhitened the $V$ and $B$ band light curves by subtracting out several dominant peaks that correspond to the long cycle periods, using the method of Vanicek (1971), as implemented in SHV. We then used least squares to find the best fit sinusoid in the residuals of the complete dataset for each filter (excluding Season 1997, with its uncertain zero points). Once the pair of rotational ephemerides were redetermined, we imposed them on the seasonal light curves and solved again for the mean amplitude and the deviation of the phase of minimum light as before. This time we found a more uniform signal with much less rapid (season to season) variations in the rotational amplitudes. However, the general trend in the amplitude variations remained the same. Figure 6 depicts the behavior of the amplitudes, starting at a level of 5-7 mmag in the early seasons, decreasing to 1-2 mmag from 2003 to 2005, and remaining at the lower level through the 2011 season.

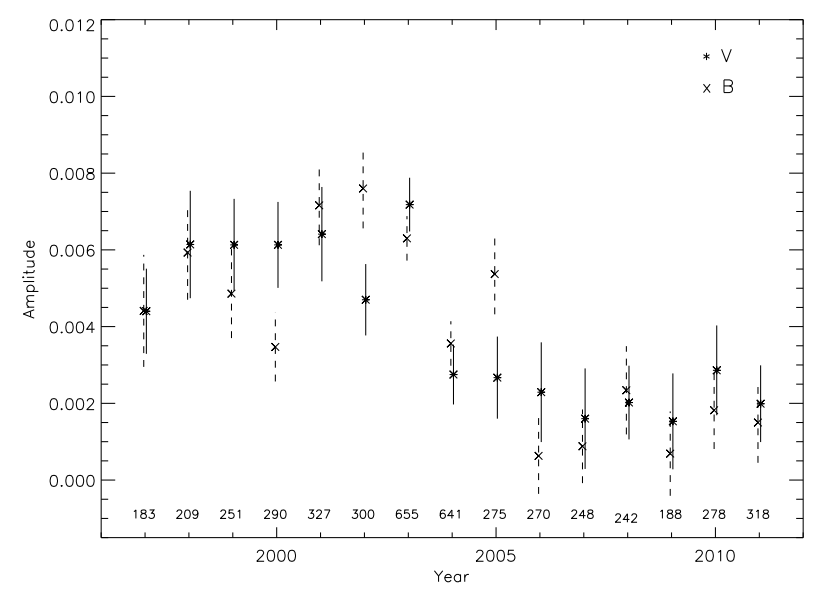

Fig. 6. - Full amplitudes of the rotational signature in $B$ and $V$ for each of the 15 observing seasons. These values were determined by rectifying the seasonal means to a common value then prewhitening the 15-year $B$ and $V$ datasets for several periods consistent with the long cycles found in various individual seasons. The numbers of $V$ observations in each season are given at the bottom of this and the following figure.

Next, recognizing that sine curves do not always give a good representation of the long cycles, we subtracted the long-cycle fits shown in SHV and in our Figs. 4 and 5 and repeated our analysis yet again. We imposed an artificial sinusoid to the full $B$ and $V$ datasets with an amplitude of $3.0 \mathrm{mmag}$ and an arbitrary control period of 1.3418644 days in place of the rotation signal. We then attempted recovery of this period by fitting the light curve of each season. Dividing the entire observing timeline into two equal segments of seven seasons (1997-2003 and 2005-2011; the omission of Season 2004 recognizes that this season seems to be transitional), we found mean amplitudes in $V$ and $B$ of 3.77 and 3.90 ( $\pm 1.5 \mathrm{mmag})$, respectively, for the first half and 3.20 and 3.24 ( $\pm 0.7 \mathrm{mmag})$ for the second. We consider 
these slight differences to be a measure of the uncertainties introduced by our processing, incomplete subtractions of long-cycle signals, and periodogram sidelobes from gaps in our observing windows.

In contrast to our experiment with a control period, results from the real-data residuals, shown in Figure7, differ rather little from those of the previous analysis of real data and show a marked contrast to the behavior of the control set. The primary difference is that the rotational amplitudes decrease smoothly during Seasons 2002-2005. For the first seven seasons, we found amplitudes averaging 5.4 and 4.8 ( $\pm 1.05 \mathrm{mmag})$ in $V$ and $B$, respectively, and for the last seven seasons 2.0 and $1.8( \pm 1.01 \mathrm{mmag})$, mean differences that are almost six times larger than the controls. Moreover, the signals in the last several seasons are nearly undetectable, leading us to realize that had we started our program a few years later, when the amplitudes had already decreased, an important facet of the $\gamma$ Cas puzzle - the discovery of the rotational modulation - might not have been unraveled for some time yet to come.

One challenge to this result might be that visible changes in the observed magnitudes that are caused by minor mass-loss episodes degrade the fit of our models, which are based on simple analytical functions. For example, if the star were more active in ejecting mass during the last seven seasons, it would brighten the star in ways that we cannot anticipate. This would degrade the fits to low-amplitude signals such as the rotational modulation, leading to lower observed amplitudes. To check for this possibility, we computed the mean and rms of the systematic differences (absolute values) for all seasons of both the $B$ and $V$ light curves. The results showed these metrics to be constant over time, with no first/second half trends apparent. Thus, there is no evidence that the long cycles introduce long-term biases in our determination of the amplitudes of the 1.21-day signal.

An interesting property of this signal is that the overall mean $r=\Delta B / \Delta V$ ratio from analysis of the residuals is $1.02 \pm 0.16$. This value is indistinguishable from unity and is also consistent with the results of SHV. This confirms that the modulation originates in a region having the same color as the surface of the Be star.

We take the following as our best ephemeris for the rotation signal:

$$
T_{\min }=R J D 53000.407 \pm 0.024+(1.215811 \pm 0.000030) \times E .
$$

where the epoch, referenced to light minimum and period are mean values computed from the several $B$ and $V$ determinations of these quantities above, and the uncertainties are the standard deviations of the means. Our new period makes only a slight improvement to $P=1.21581 \pm 0.00004$ days from SHV, probably because the amplitudes were significantly lower in our new observations. 


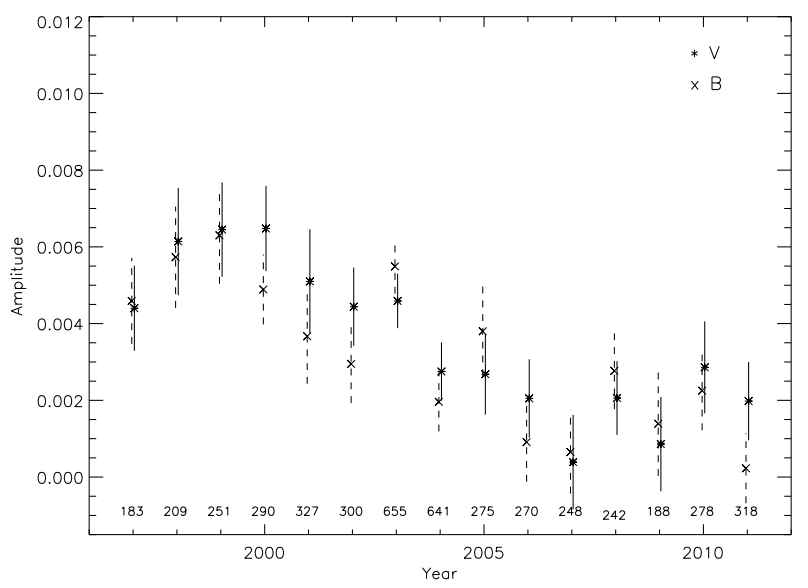

Fig. 7.- Full amplitudes of the rotational signature in $B$ and $V$ for the 15 individual seasons in our study. These values were determined from the light-curve residuals after removing the long-cycle modulations found in SHV and in Fig.4.

To show the brightness variability in detail, we plot in Figure 8 the $V$ data residuals from the long cycles for six successive nights from 2003 November 19-24 against the mean period. This short subset is ideal for analysis because the long cycle has all but damped out during this period. Moreover, the data coverage on these nights is the densest we obtained for any consecutive nights in the entire program. Light curves for both passbands are exhibited in SHV. Figure 8 confirms the nonsinusoidal waveform found by SHV. Its shape is reminiscent of an asymmetric radial-velocity curve of a binary system. Therefore, we fit the data with a Lehmann-Filhes-type solution of the form:

$$
m=K \cos (\phi+\omega)+e \cos (\omega)
$$

where we used the period and time of a fiducial phase to determine the rotation phase $\phi$ and a semiamplitude $\mathrm{K}=1.43 \mathrm{mmag}$ from procedures described just below. This left three free parameters that were needed to model the waveform, a semiamplitude of $0.0076 \pm 0.0010$ mmag, a pseudo "eccentricity" $e=0.51 \pm 0.05$, and a "longitude of periastron" $\omega=25^{\circ} \pm 6^{\circ}$; the full amplitude of course is $2 \mathrm{~K}$. Similar values were found for the $B$-band dataset. The formal $r m s$ is 0.0040 , which is in the range of 0.0035-0.004 we had already found for the nightly errors (SHV). Using this value in our Lehman-Filhes fitting gives a reduced $\chi^{2}$ statistic is 1.01 (206 degrees of freedom), which is essentially a consistency check on the input rms value. The corresponding reduced $\chi^{2}$ for a sinusoid of the same amplitude is 1.34. As an independent check of our values, we rereduced the data for this figure by using the computer 
program BISP written by Dr. Frank Fekel that uses a modified form of the Wilsing-Russell method (Wolfe, Horak, \& Storer 1967), followed by a differential corrections program, SB1, by Barker, Evans, \& Laing (1967). This effort resulted in an eccentricity of $e=0.53 \pm 0.05$ and an $\omega=6^{\circ} \pm 6^{\circ}$. The analysis of the $B$ band data gave similar results. Note that together the $e$ and $\omega$ represent "bowness" and "asymmetry" attributes of the waveform. They are not independent and hence orthogonal parameters. For $e$ and $\omega$, the quoted errors were determined from the different values of these parameters from the individual $V$ and $B$ band light curves. Also, the observational errors for the $V$ and $B$ band datasets are likely highly correlated, so this is still not a satisfactory test for significance.

To address this issue of the statistical significance that the waveform is nonsinusoidal, we investigated the chances that a sine curve with the given noise properties could give a value of $e$ similar to 0.53 that we found. We subjected this result to a more formal yet still conservative test by checking on the significance of the "eccentricity" implied in the data and ignoring the asymmetry of the light curve, that is the $\cos (\omega)$ component of the e $\cos (\omega)$ term. We began by forcing the solution of our data to a sine curve, that is by adopting the previous period, mean light level, and amplitude and running the LehmanFilhes program once again. This gave a somewhat higher reduced chi-squares value of 1.34. We then ran simulations on the best sine curve fit with the rms value we had found in our original solution, allowing the $e$ parameter to be floating once again. We found that it took 1.7 million realizations for the $e$ value to reach our measured value of 0.53 , suggesting that the nonsinusoidal eccentricity parameter is significant at a value of $5 \sigma$. Running the test once again with a new initial seed value for the random number generator gave almost the identical results. Meanwhile, we found that the lower bounds of our computed mean $\chi^{2}$ values in our simulations attained the value we found from our full-parameter Lehman-Filhes solution of our data at a slightly lower significance level of $4 \sigma$. In view of these tests we conclude that the waveform was significantly different from a sinusoid, and also shaded in a different direction from the right-shaded asymmetric waveform solved for by SHV over the 1997-2004 seasons. These conclusions are also consistent with the annual meandering of the fitted phase of light minimum (again to a significance of several times our formal errors that result from our analysis of the changes in light amplitude described above.

\subsection{A search for other periods}

Finally, we searched for the influence of the secondary star on the light curve through its known orbital period of 203.5 days. Our main concern initially was to mitigate the effects of artificial low frequencies, principally in the seasonal window. We found that the effect of 


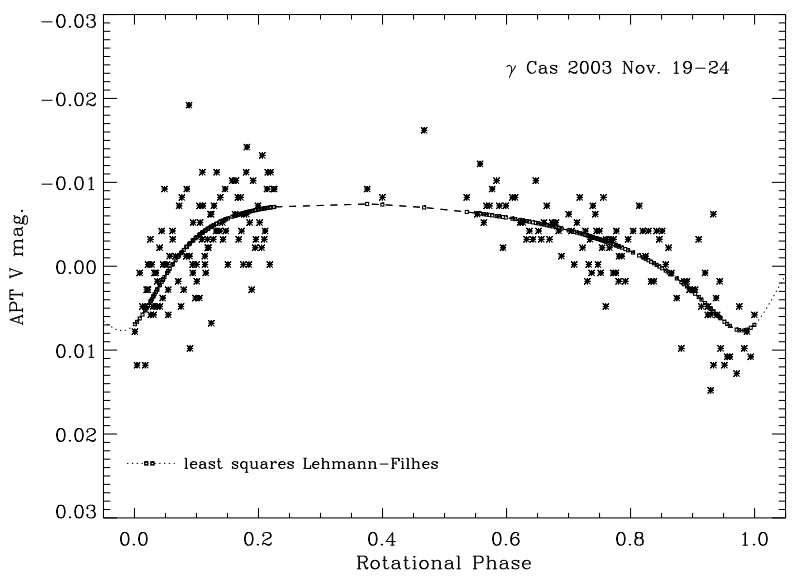

Fig. 8.- Light curve of the $V$ residuals for 2003 November 19-24 (RJD 52962-52967) phased to a period of 1.21581 days. Symbols refer to observations taken on different nights during this interval. The solid line is the fit from a least-squares Lehmann-Filhes solution $\left(e=0.49, \omega=40^{\circ}\right)$.

the lunar synodic period on the local sky was insignificant. Other studies using the T3 APT have found the same null result. The procedure that worked best was to "condition" the data first by removing obvious $(3 \sigma)$ outliers and also removing the first observing season due to its zeropoint uncertainty. The resulting "raw" Fourier periodogram showed a dominant broad period centered at 351 days. We attribute this to the interseason gaps associated with the Earth's orbital motion. A clutter of sidelobes due to the combinational frequencies of this and the diurnal frequency are present below $0.04 \mathrm{cy} \mathrm{d}^{-1}$. Most of the signal from this noise was mitigated by detrending of the 13 seasons of data. The remaining (mainly combinational frequency) lobes were equivalent to signals at a level of $\approx 5-10$ mmags. We inspected the resulting periodogram and found a 3 mmag signal due to the rotational modulation discussed above. The low frequency range was dominated by a few weak signals associated with an 80 day period (from Seasons $2003 \&$ 2007) and other incompletely removed cycles. However, we found no signal whatsoever at $0.0050 \mathrm{cy} \mathrm{d}^{-1}$, associated with 203.5 days or at any of its precise harmonics. We then injected a series of sinusoidal signals of diminishing amplitudes of this frequency and attempted to recover them through their periodograms. We discovered that we could recognize an orbital frequency signature down to $2.5 \mathrm{mmag}$ (about the level of the rotational signal). However, we were unable to recover the amplitudes below 5 mmags. Figure 9 shows the results of these simulations. Below 5 mmags., the recovery becomes nonlinear and unpredictable. We take this value as the 2 sigma limit against the detection of a full-amplitude, orbitally-induced signal. 


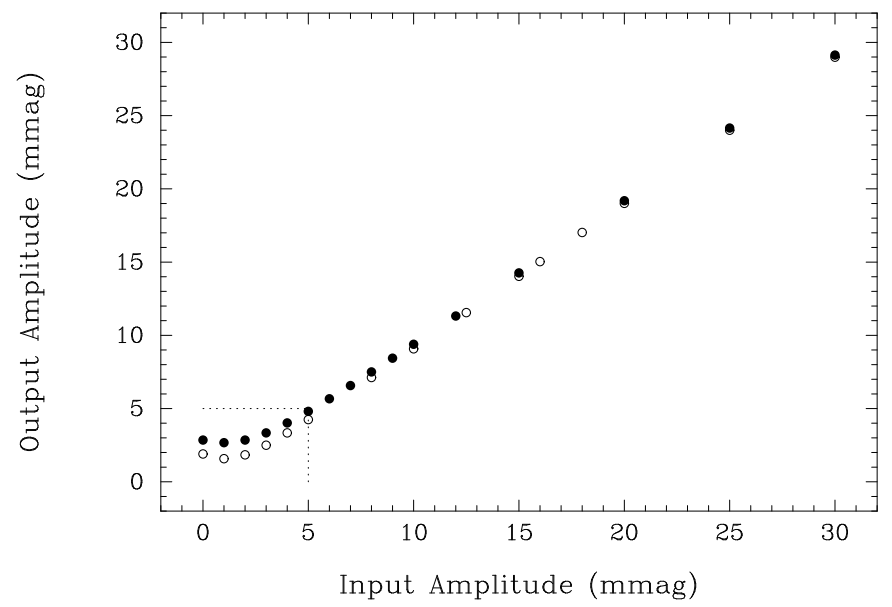

Fig. 9.- The relation between artificial input signals at the orbital frequency in our noisy light curves and their recoveries by periodogram analysis. The recovery is linear and hence reliable to a level of $\approx 5$ mmags. (faint dotted lines).

\section{Discussion}

\subsection{The outburst, long cycles and dynamos}

Several important results have emerged from this study, and we start by discussing the long cycles, which were the first to be discovered by APT photometry. First, we have found no season in which the light curve does not betray some semblance of a cycle. Second, although these oscillations are ubiquitous, they are not stable. More often than not, the cycle lengths vary from year to year. As near as we can determine, the period continuity and phasing are unaffected when a new cycle is triggered and grows. For example, we remind the reader of the peculiar event in the 2003 cycle in which an 80 day cycle continued at first from the previous year, quickly damped out, and grew again to a new 80-day cycle with the same phasing (SHV). Thus, it may be more accurate to describe the cycles as originating from an excitation event in the disk, which damps out within one or two cycles. As it weakens, the process begins anew as another cycle is excited on a timescale of as short as two weeks (SHV).

Third, we noticed in $\$ 3.2$ that the fit for Season 2010 is poor (Fig. (4). This is just when the mass input from the star was extraordinarily high, according to the X-ray results of S12. We believe that the fit to the long cycles is flawed at this time because of a sudden and temporary change in this loss rate rather than an interruption in the disk cycle per se. A temporary reduction of the rate of the ongoing mass loss to the disk could also be responsible for the decreased brightness during the middle of Season 2005 as well. This conjecture is 
supported by the absence of the effect of mass-loss events on the cycle length or magnitude range ratio at other times. Only the rate of brightening is affected.

The S12 study was indeed fortunate to observe $\gamma$ Cas spectroscopically with $X M M$ Newton just as the star's outburst began. This study recorded the effect of the mass loss incident on one of two X-ray absorption columns. This column covered $\frac{1}{4}$ of the X-ray emitting sources and doubled in 44 days. (The second column remained constant.) Furthermore, during this time the column attained a historical high value of $7.4 \times 10^{23}$ atoms cm$^{-2}$, which was some 300 times higher than the historical minimum column length observed. The significance is that the X-ray emission sites fall along the column to the source of this absorption, the Be star. We have already noted that the concurrent cycle did not change except for the waning and waxing of its amplitude during the 2010 and 2011 seasons. This is strong additional evidence that the long optical cycles arise in the disk.

An additional marginally significant characteristic of the cycles is their potential dichotomy into two distinct populations that are defined by the gap in the $\Delta \mathrm{B} / \Delta \mathrm{V}$ histogram (Fig. (3). This implied bimodality could be important because it shows that the variations in the shifts in mean color $r$ convey physical information about the origin of the long cycles. Our interpretation of this possibility is that the cycle is excited in at least two somewhat different volumes. When they first suggested that the cycles originate within the disk, RSH could not be sure of even this interpretation. The shifts in color are a strong indication indeed that long cycles do not arise from the star's surface. We should also remark that earlier concepts of the temperature stratification of disks assumed that these regions would be segregated simply by disk radius. However, recent models of Be disks (e.g., Sigut, McGill, \& Jones 2009) suggest that the denser regions of the disk cool from recombination processes. Since this cooling is proportional to electron density squared, a cooler emitting component in the disk plane is differentiated from "warm sheaths" at some distance from the central plane. A bimodality in the color ratio may be caused by a preference of cycles being excited either in the disk plane or at higher latitudes or, alternatively, at smaller versus larger disk radii.

RSH and SHV speculated that the cycles arise from a disk dynamo, specifically via the Magnetorotational Instability ("MRI"; Balbus \& Hawley 1991, 1998). This mechanism is predicted to operate in any stellar disk isolated from external influences as long as an internal seed magnetic field exists and it undergoes Keplerian rotation. The mechanism operates by a positive feedback loop relying on the existence of a random perturbation that forces disk matter to move radially and exchange angular momentum with its surroundings. The angular velocity shearing and the seed field excites a local turbulence. These velocities amplify the initial seed field, causing an reamplification of turbulence, and so on. In the RSH 
picture, the positive feedback loop creates a dynamo in which disk zones alternately undergo matter compression and rarefactions during the cycle. The more highly compressed regions have higher electron densities and therefore higher emission measures, which is measured as light variations in the $V$ and $B$ passbands. For this MRI mechanism to be observable it must be unstable globally. In general, organization of modulating MRI cells cannot be predicted. Furthermore, if the magnetic field from the star intrudes into the disk, it will quench the mechanism. We note also that simple arguments suggest that the cycle length should be of the order of the orbital period of particles in the disk - a few days. Nonetheless, the disparity between this value and the observed lengths, $2-3$ months, is itself no disqualification since, for example, even the length of the solar 22-year cycle has yet to be predicted.

Putting aside for a moment these obvious issues, the existence of a mechanism that segregates different constituents of the gas disk offers the possibility of changes from time to time in those regions where the MRI operates most efficiently. To take one example, Emmanuel \& Balbus (2012) suggest that diffusion of dust grains toward the central plane of protoplanetary disks can create a "dead zone" that suppresses the MRI. We cannot suggest the creation of grains in a Be star's disk. However, one can imagine more realistic scenarios. Perhaps higher than usual turbulent velocities in the less ionized central plane, where the gas is already more neutral, can decouple gas from the local field. Then, by limiting the MRI to operate in warmer (sheath) volumes only where the disk's contribution to the $B$ flux is greatest, the brightness range ratio $\Delta B / \Delta V$ would increase during the cycle. Such reasons could in principle give a range in the values of this color ratio over the a number of cycles. Clearly this subject is highly speculative.

\subsection{The rotational modulation feature}

In this paper we have again recovered the 1.21 day period found by SHV and confirmed that it is robust over the full data span. Also, it undergoes no color changes during the period. SHV presented reasons why the feature is rotational, among them that the expected stellar radius (from HR Diagram and interferometry) and measured values of $v \sin i$ and $\sin i$ (interferometry) predict a period of $1.25 \pm 0.17$ days. At the time SHV analyzed their results, both empirical results and models suggested that it seemed unlikely that B0.5 V stars would excite NRP modes with periods near 1 day. In the intervening years the launch of the CoRoT satellite (Baglin et al. 2006) and the bonanza of data it generated in fact has led to the discovery of NRP $g$ mode periods near 1 day in O9-B1.5 III-V stars (Degroote et al. 2009, Papics et al. 2011, Briquet et al. 2011), even though the excitation mechanism and the predicted properties of these modes are still unclear (possibly because current internal 
opacity models are still inadequate). When the data sampling intervals are long and the signal to noise ratios are high enough, several modes are invariably discovered with periods from the $\beta$ Cephei star range of a few hours up to about 1.5 days. Analysis of periodogram features in the light curve of HD 180642 indicates that the amplitudes of some modes change, while others are stable over timescales of up to three years (Degroote 2009). However, these stars differ from $\gamma$ Cas by being slow to intermediate rotators, whereas $\gamma$ Cas's rotation rate is almost indistinguishable from the critical velocity.

In fact, despite the new work, no NRP modes with periods near an expected rotational period have been found in these stars. This is not surprising because most of the periods in the corotating frame of the star would then be very long - indeed, within the errors of their determinations, nearly infinite. However, it is possible in principle for very long periods to be observed if a mode exists with a period that is long, but still somewhat shorter in the nonrotating frame, and if it is accompanied by a retrograde $(m>0)$ with a large rotational splitting. In principle, such splitting could give a frequency mode component that compensates for the positive frequency of its unsplit component mode $(m=0)$, again in the nonrotating frame. In this scenario only this one retrograde mode would be detected. While such a thought experiment is possible, we regard it as ad hoc and not very likely. Moreover, we have seen that this periodicity exhibits no color term and no measureable phase difference between the $B$ and $V$ fluxes. In contrast, NRP $g$ modes of high degree and low frequencies in the corotating frame have long been predicted and are well known to exhibit color changes over their cycles. This is contrary to what we observe for $\gamma$ Cas. Based on this fact and on the unlikelihood of discovering a mode whose frequency in the initial frame must be very close to the rotational frequency, we reject the NRP hypothesis as the cause of the photometric 1.21 day signal. Finally, as listed just below, there are independent reasons for believing that disorganized surface magnetic fields exist on this star, and these could lead to this signature.

The most important new results in this paper are that the amplitude of the surface modulation decreased from 2003 to 2005, and the feature has been barely detectable ever since. The occurrence of the 2010 outburst showed no correlation with the properties of this feature. We have also discovered that the "faint star" phase can vary significantly, as it did in 2003. This circumstance was traced to a change in the asymmetry of the waveform. Then, contrary to SHV's impression that rapid optical variations are influenced by simultaneous changes in local X-ray flux over several hours, we now see that the optical brightness variations on this and other nights are due mainly to rotational modulation. From the present ephemeris, we find that the two major UV continuum dips and X-ray excesses observed by the HST and RXTE occurred at phases 0.45 (lesser UVC dip) and 0.78 (deeper dip). According to the asymmetric waveform for this epoch (SHV), these phases correspond 
to a point on the brightening branch of the light curve and another just after brightness maximum.

Pertinent to our rotational analysis of $\gamma$ Cas is the detection of rotational modulation signatures in other hot stars for which magnetic fields are not known to exist, either because they are below the level of detection or have not been searched for. In recent years, rotational signals have been found in light curves of HgMn stars such as $\alpha$ And and AR Aur (e.g., Wade et al. 2006, Folsom et al. 2010). Analysis of Doppler imaging results for a number of HgMn stars, which are slow rotators $\left(v \sin i \approx 70 \mathrm{~km} \mathrm{~s}^{-1}\right.$ ), indicates that various elements on the surfaces of these stars are distributed inhomogeneously, that is, in discrete spots. Spectropolarimetric observations of 36 of these stars by Makaganiuk et al. (2011) have revealed no attendant magnetic field signatures. These authors advance field/gas energy partition to argue that magnetic field configurations in these spots cannot hide these fields from detection and therefore that the spots have a nonmagnetic origin. Rather, they speculate that spots arise from a yet undiscovered hydrodynamic instability that augments diffusion timescales locally by several orders of magnitude in a nonmagnetic environment. However, in the present application it appears that no argument can be reasonably advanced that these hypothetical effects create surface structures on more massive and rapidly rotating stars.

Two other recent studies have found rotational modulation in main sequence B stars. First, Papics et al. (2001) have discussed variability of light curves of HD 51756 obtained by the CoRoT satellite. This is a double-lined binary system of which the primary is a B0.5 IV $\operatorname{star}\left(v \sin i=170 \mathrm{~km} \mathrm{~s}^{-1}\right)$ and the secondary is an early-type $\operatorname{star}\left(v \sin i=28 \mathrm{~km}^{-1} \mathrm{~s}^{-1}\right)$. The light curve displays millimag level variabilities. According to these authors, the variations must be due to harmonic structure of a single frequency, probably rotational modulation, and not to nonradial pulsations. The variations can then be understood as being caused by a single surface disturbance, with $i \approx 41.6^{\circ}$ if they arise from the primary or with $i \sim 6.3^{\circ}$ if they come from the secondary. Because magnetic measurements of this star have not yet been attempted, Papics et al. were not able to discuss the physical origin of the rotational modulation. Interestingly, if it should be determined that the source of the modulation is on the primary, it would require the star to be rotating at near the critical velocity. A reference to the Rossi All Sky Survey shows no strong X-ray source that could be identified with this system.

A second study showing rotational modulation in a normal abundance B star is that of McNamara, Jackiewicz, \& McKeever (2012). These authors obtained precise light curves of the star KIC 005473826 (a likely late-type B star) using Kepler spacecraft data and found two frequencies. The primary signal occurs at $0.95 \mathrm{~d}^{-1}$. A second frequency twice this value 
is likely to be its harmonic. The amplitudes of these two frequencies are 10.8 mmags and 6.0 mmags, respectively, i.e., they are larger than we have found for $\gamma$ Cas. These authors ascribe the signal to rotational modulation of a magnetic spot. This star seems to be beyond the reach of present spectropolarimetric systems for a direct magnetic detection.

We conclude this discussion with a summary of arguments for surface magnetic fields on the surface of the Be star $\gamma$ Cas apart from the rotational surface feature. Each of the following is an independent argument for the existence of matter being contained in a volume situated above one or more regions of the Be star's surface:

1. the existence of the $m s f$ moving rapidly across optical and UV line profiles in the star's spectrum. This implies the corotation of small, short-lived cloudlets (Smith, Robinson, \& Hatzes 1998, "SRH"; Smith \& Robinson 1999, "SR").

2. the above mentioned brief dips over several hours in the UV continuum, indicating the existence of 3-4 optically thin, corotating clouds. Other dips have been found in light curves formed from contemporaneous IUE data (SRH). Surface starspot models can be ruled out from their rapid evolution of the transits in time and from the redness of their color in the UV wavelength domain (SRH).

3. the existence of corotating clouds having unexpected temperatures near the surface of a B0.5 star. Simultaneous observations suggest that the ionization of Fe and Si in regions over the star responded to changes in X-ray flux (Smith \& Robinson 2003).

Each of these arguments supports the presence of circumstellar matter close to but not on the stellar surface, unlike the rotational feature.

The evidence for magnetic fields being responsible for the spots is quite strong. The fact that they are likely to be disordered and to have decreased in the last few years at least partially addresses the concern of Neiner et al. (2012) that a spectropolarimetric observation during this period did not lead to a positive result. Depending on the complexity of their multipolar structure, it can be hoped that future observations can discover them when the amplitude of the photometric modulation recovers to its its former value. It is important to point out in any case that such fields must necessarily have complex toplogies, since they would otherwise be easily inferred from Bp signatures (cyclical emission/absorptions in the UV resonance lines of $\mathrm{Si}, \mathrm{C}$, and N) that accompany dipolar fields. 


\section{Conclusions}

For over two decades, the overriding question concerning $\gamma$ Cas has been the mechanism behind the production of its hard X-rays. Ironically, it is possible that the ultimate resolution of this question will not come so much from continued X-ray observations of the $\gamma$ Cas stars as much as traditional optical studies. First, radial velocities must be derived to determine whether binarity is a necessity for the $\gamma$ Cas-like X-ray properties to develop, and, if so, what the orbital separations and eccentricities are - such parameters are relevant to the determination of the X-ray flux generated by accretion of mass by the secondary. Their role in binaries may also determine why they reside in such a small region of the HR Diagram and, indeed, whether this determines a particular type of evolved secondary.

Second, and pertinent to this study, is the role of traditional optical light monitoring. Despite attempts to start such work on the next brightest star, HD 110432, such work has not yet taken root in the Southern hemisphere. Even so, there is a great deal of work that could be done to characterize its optical variations. If this star also shows rotational variability, the $v \sin i$ suggests the rotational period is also near one day. This is clearly a task best suited to an APT or space-borne telescope.

Our $\gamma$ Cas campaign has led to several unanticipated discoveries pertinent to the X-ray excitation mechanism. These include:

1. long optical cycles, probably often correlated with XR cycles (RSH, SHV),

2. optical brightenings/reddening in the disk correlated with attenuation of XR cycles,

3. partial damping of long cycle amplitudes due to the mass loss events,

4. cycles exciting in somewhat different regions of the disk,

5. rotational signal and waveform varies over time.

Concerning this last point, the extended and asymmetric minimum appearing in the SHV solution of the average for Seasons 1997-2004 probably arises from a superposition of changing waveforms during this period. This means that whereas the centroid of the surface feature that causes this is stable, the internal distribution of the disturbing property, assumed to be due to magnetic fields, is not. Moreover, the "sharpness" or even the "asymmetry" cannot be considered a permanent and defining characteristic of the light curve.

We noted in 4.2 that the UV continuum dips and X-ray excesses observed by SRH coincide with APT rotational phases 0.45 and 0.78, i.e., shortly before brightness maximum and near the brightness null. This phasing suggests one of two possible explanations. 
The first is the mechanism producing rotational modulation in this B0.5e star is fundamentally different from those of later B-type stars - that is, a magnetic structure causes a local optical brightening. Support for this view comes from the discovery in visible-band light curves of two magnetic Of?p stars, HD 191612 and HD 108, that maximum brightness coincides with the transits of magnetic spots and maximum $\mathrm{H} \alpha$ emission (Holwarth et al. 2009; Barannikov 2007; Martins et al. 2010). A theoretical construct for bright magnetic spots has been advanced by Cantiello \& Braithwaite (2011). Their argument is that magnetic pressure in the spots partially offsets gas pressure once pressure equilibrium is established, and this reduces the radiative opacity along a column. The lower opacity allows photons to escape from a deeper, hotter and, therefore, brighter photospheric region than would be the case in a nonmagnetic atmosphere. (This is essentially the reason that faculae appear bright above thin magnetic tubes on the solar surface.) According to these authors, these arguments should apply for stellar masses down to about $10 \mathrm{M}_{\odot}$. However, for the magnetic Bp stars (i.e., for masses up to $9 \mathrm{M}_{\odot}$, or type B2), it is known that the optical flux decreases during the transits of magnetic spots. Then for these stars the physical explanation must be altogether different. In Bp star light curves, periodic decreases in optical flux are caused by the appearances in the optical continuum of bound-free absorption edges caused by superabundant heavy elements as chemically peculiar spots (approximately coinciding with magnetic spots) transit the star's surface. Given an assumed mass of $14-15 \mathrm{M}_{\odot}$ (e.g., Harmanec 2002; Gies et al. 2007), it seems on the face of it that the explanation for the magnetic O star variability could also hold for $\gamma$ Cas.2

A second, perhaps equally compelling, interpretation is that the inferred, active X-ray center/cloud complexes observed in 1996 January in $\gamma$ Cas do not reside on fixed locations at all. In this explanation the clouds producing those UV dips were transient and occurred at no special longitudes on the Be star's surface. This carries the important implication that the circumstellar clouds causing them and therefore the underlying X-ray active structures, do not remain at fixed longitudes. In fact, in the RS scenario X-rays are produced by surface impacts of high energy electron beams originating from external magnetic field relaxations. If these beams are not channeled to preferred surface locations by open field lines anchored to permanent spots, the ensuing flare events might well occur at essentially random areas on the Be star. In this case, the current bright-star alignment with the X-ray active spots in 1996 was coincidental.

Going forward, we emphasize that studies of $\gamma$ Cas and other analogs must not only define their properties as a group but even more importantly, they must also determine what

\footnotetext{
${ }^{2} \mathrm{SHV}$ attempted to tie the rotational signature of $\gamma$ Cas to the phenomenology of other magnetic Bp stars like HD 37776. The current discussion should correct their speculation on this point.
} 
sets them apart from other B0.5-B1e stars that rotate very rapidly and yet do not show abnormal $X$-ray emissions. In addition, having been building now for several decades, perhaps the disk of $\gamma$ Cas will start to decay within a few years. It will then become critical to find out whether the emission continues when the disk decays but is still strong. In this circumstance, the disk could no longer plausibly transfer mass through resonance interactions with its companion, and the last plausible efficient conduit of matter transfer to a degenerate companion will be lost. This will provide an important test of whether X-rays are generated via blob accretion onto a white dwarf. Thus, the coming goals of a continuing APT monitoring program will be to await both the end of the contemporary disk building era and (independently) the restoration of the rotational modulation to its former strength. The latter event will offer a better chance of discovery of a magnetic field by current spectropolarimetric techniques.

The authors would like to express their appreciation to the referee for valuable scientific comments that improved this paper. We also profited from the use of two orbital-velocity solution codes written by Dr. Frank Fekel. GWH acknowledges long-term support from NASA, NSF, Tennessee State University, and the State of Tennessee through its Centers of Excellence program. MAS acknowldedges funding of NASA Grant NNX11AF71G through the to Catholic University of America under the Advanced Data Analysis Program.

\section{REFERENCES}

Baglin, A., Auvergne, M., Barge, P., et al. 2006, in Special Publ. ed. M. Fridland, A. Baglin, 1306, 33

Balbus, S., \& Hawley, J. F. 1991, ApJ, 376, 214B

Balbus, S., \& Hawley, J. F. 1998, Rev. Mod. Phys., 70, 1B

Barker, E. S., Evans, D. S., \& Laing, J. D. 1967, R. Obs. Bull. 130

Barranikov, A. A. 2007, Inf. Bull. Var. Stars, 5756, 1

Barylak, M., \& Doazon, V. 1986, A\&A, 159, 65B

Briquet, M., Aerts, C., Baglin, A., et al. 2011, A\&A, 527, 112B

Cameron-Collier, A., \& Robinson, R. D. 1989, MNRAS, 236, 57C

Cantiello, M., \& Braithwaite, J. 2011, A\&A, 534, A140

Degroote, P., Briquet, M., Catala, C. 2009, A\&A, 506, 111D

Eaton, J. A., Henry, G. W., \& Fekel, F. C. 2003, in The Future of Small Telescopes in the New Millennium, Vol. II, The Telescopes We Use, ed. T. D. Oswalt (Dordrecht: Kluwer), 189E 
Eaton, J. A., \& Williamson, M. H. 2007, PASP, 119, 886E

Folsom, C. P., Kochukhov, O., et al. 2010, MNRAS, 407, 2383F

Gies, D. R., Bagnuolo, W. G., Baines, E. K., et al. 2007, ApJ, 654, 527G

Güdel, M., \& Nazé, Y. 2009, A\&ARv, 17, 309G

Harmanec, P., Horn, J., Koubsky, P., et al. 1980, BAIC, 31, 144H

Harmanec, P. 2002, Exotic Stars as Challenges to Evolution, ed. C. A. Tout \& W. van Hamme, ASP Conf. Ser., 279, 221H

Henry, G. W. 1995a, in ASP Conf. Ser. 79, Robotic Telescopes: Current Capabilities, Present Developments, and Future Prospects for Automated Astronomy, ed. G. W. Henry \& J. A. Eaton (San Francisco: ASP), 37H

Henry, G. W. 1995b, in ASP Conf. Ser. 79, Robotic Telescopes: Current Capabilities, Present Developments, and Future Prospects for Automated Astronomy, ed. G. W. Henry \& J. A. Eaton (San Francisco: ASP), 44H

Hirata, R. 1982, in Be Stars, IAU Symp. No. 89 (Dordrecht: Reidel Publ.), p. 41

Hirata, R. 1995, PASJ, 47, 195H

Jernigan, J. 1976, IAU Circ. 2900

Lopes de Oliveira, R., Motch, C., Negueruela, I., et al. 2006, Adv. Sp. Res., 38, 2782L

Lopes de Oliveira, R., Smith, M. A., \& Motch, C. 2010, A\&A, 512A, 22L

Makaganiuk, V., Kochukhov, O., Piskunov, N., et al. 2011, A\&A, 525A, 97M

Marco, A., Motch, C., Ribó, M., et al. 2009, AdSpR, 44, 348M

Markwardt, C. B. 2011, Markwardt IDL Library, http://www.physics.wisc.edu/ craigm/idl/idl.html

Martins, F., Donati, J.-F., Marcolino, W. L., et al. 2010, MNRAS, 407, 1423M

McNamara, B. J., Jackiewicz, J., \& McKeever, J. 2012, AJ, 143, 101M

Neiner, C., Grunhut, J. H., Petit, V., et al. 2012, MNRAS, in press

Okazaki, A. T., \& Negueruela, I. 2001, A\&A, 377, 1610

Papics, I., Briquet, M., Auverge, M., et al. 2011, A\&A, 528A, 123P

Peters, G. P. 1982, PASP, 94, 157P

Porter, J. M., \& Rivinius, T. 2003, PASP, 115, 1153P

Robinson, R. D., \& Smith, M. A. 2000, ApJ, 540, 474R (RS)

Robinson, R. D., Smith, M. A., \& Henry, G. W. 2002, ApJ, 575, 435R (RSH) 
Secchi, A. 1866, Astron. Nachr., 68, 63

Sigut, T. A., McGill, M. M., \& Jones, C. E. 2009, ApJ, 699, 1973S

Slettebak, A., \& Snow, T. P. 1978, ApJ, 224, L927

Smith, M. A. 1995, ApJ, 442, 812S

Smith, M. A., \& Balona, L. B. 2006, ApJ, 640, 491S

Smith, M. A., Henry, G. W., \& Vishniac, E. 2006, ApJ, 647, 1375S (SHV)

Smith, M. A., Lopes de Oliveira, R., et al. 2012, 540A, 53S (S12)

Smith, M. A., \& Robinson, R. D. 1999, ApJ, 517, 866S

Smith, M. A., \& Robinson, R. D. 2003, Interplay of Periodic, Cyclic, \& Stochastic Variability in Selected Areas of the H-R Diagram, ed. C. Sterken, ASP Conf. Ser. 292, 263S

Smith, M. A., Robinson, R. D., \& Corbet, R. H. D. 1998, ApJ, 503, 877S (SRC98)

Smith, M. A., Robinson, R. D., \& Henry, G. W. 2002, ApJ, 575, 435S

Stee, P., \& Mourard, D., Bendjoya, Ph., et al. 2012, A. \& A., in press

Vaniĉek, P. 1971, Ap\&SS, 12, 10V

Wade, G. A., Aurière, M., et al. 2006, A \& A, 451, 293W

White, N. E., Swank, J., et al. 1982, ApJ, 263, 277W

Wolfe, R. H., Horak, H. G., \& Storer, N. W. 1967, in M. Hack (ed.), Modern Astrophysics, Gordon \& Breach, New York, p. 251

Yang, S., Ninkov, Z., \& Walker, G. A. H. 1988, PASP, 100, 233Y

This preprint was prepared with the AAS IATEX macros v5.0. 